\title{
EBD Podcast Series - excerpts from an interview with Dr Jane Gillette
}

\author{
Dominic Hurst \\ Centre for Evidence-based Dentistry, Oxford, UK
}

This is an abridged transcript of an interview with Dr Jane Gillette conducted on 19/11/2010. The full interview can be accessed at http://www.nature.com/ebd/indexpod.html.

Jane is a clinical research dentist and private practitioner. She sits on the executive board of the NW PRECEDENT practice based research network, one of three federally funded practice research networks in the USA. Jane is also section editor for general dentistry at the Journal of Evidence-Based Dental Practice.

\section{On her current involvement in evidence-based dentistry:}

I'm focusing on assisting other dentists with understanding the principles of evidence-based dentistry, on literature searches, critical appraisal, how to use resources such as MOOSE [Meta-analysis of Observational Studies in Epidemiology] guidelines or AMSTAR [Assessment of Multiple Systematic Reviews] guidelines.

For some people it's helping them to understand how to form a clinical question... some people are ready to learn different ways to manoeuvre through Pubmed, to find systematic reviews and meta-analysis... some I assist with understanding how to critically appraise evidence...

I'm working with the American Dental Association (ADA) on producing You-Tube-like videos that will be published on the ADA's EBD website to help practitioners understand more about EBD and also how to critically appraise evidence.

I'm [also] interested in how to train support staff, because I think sometimes practitioners are so busy they don't really have time to dedicate to doing literature searches and evaluating the level of evidence.

[I am also] working with practice-based research networks, specifically the one that I'm involved with is the NW PRECEDENT research network, which is one of the three research networks across the US that are funded by the National Institute of Health/NIDCR [National Institute of Dental and Craniofacial Research].

\section{On the role of support staff in bringing EBD into}

the practice:

In my office, everyone gets [an EBD] textbook. They complete each section then the worksheets associated with each section and then they turn it in to me. We review their answers from the worksheets and talk about the principles that they've learnt in that chapter on that topic and after they complete that section they receive $\$ 100$. As their final project they pick the topic that's interesting to them and do a review of the literature. They ask a clinical question. They find the evidence and then they critically appraise that evidence and they share it with our group. One of the most important changes I've seen in my practice since I've been doing that is how on fire and articulate my own staff are about evidence-based dentistry or what's unique about the practice we're in.
On what got her started in EBD:

One was a class that I took in my fourth year of dental school [on EBD]. I'm quite a bit of a sceptic and I really liked the idea of being able to answer the questions myself. I really started to apply it when

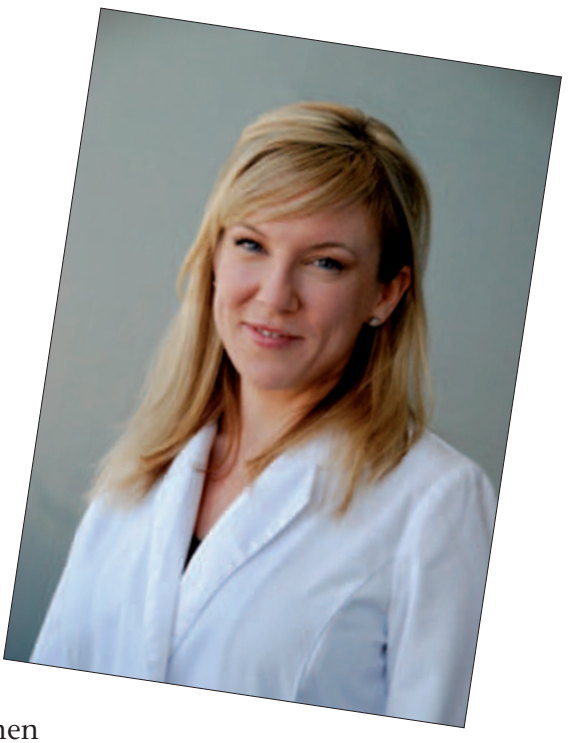

I was working in a community clinic, a federally-qualified health centre. I had to implement a couple of key protocols in my office that were going to impact on the disease rate and improve the health of my patients. I sat down and went through the literature. [I] started with the systematic reviews and then moved out from there and sorted it out by topic. Once I found all that information [I] started to critically appraise that evidence and then decided which protocols to implement in the practice.

On the challenges of introducing EBD into teaching, clinical practice and to patients:

One is the time - how to find time in an already busy and stressful lifestyle to do [EBD] and then also how do you communicate evidence-based dentistry to your patients. How do you tell them what's different? When [my staff] take telephone calls for new patients they introduce the concept of evidence-based dentistry right away. They'll say 'Dr Gillette practises evidence-based dentistry and what that means is that she makes decisions of care based upon the best scientific evidence and not based on tradition.' I think professionally, teaching, one of the biggest problems is that sometimes there's a lack of quality literature to look at to answer your question. And then you're back to the basic principles of using your own professional expertise and the patient's values and desires.

\section{On changes to her work environment and EBD}

more generally:

In my workplace and practice I'm working on standard operating procedures that are based on evidence that has been graded. I think that all journals should be required to grade the level of evidence that they're publishing. That's a big request but there are some medical journals that already do that and of course the Journal of EvidenceBased Dental Practice and Evidence Based Dentistry already do.

Our thanks go to Jane for taking the time to share her experiences and thoughts relating to EBD.

Evidence-Based Dentistry (2011) 12, 4. doi:10.1038/sj.ebd.6400768 